\title{
Protective effect of BCG vaccination in infant Asians: a case-control study
}

\author{
G E PACKE AND J A INNES
}

Birmingham Chest Clinic, Birmingham

SUMMARY BCG vaccination has been routinely offered to infant Asian children in Birmingham since 1965. We conducted a case-control study to assess the protective effect of this scheme. Altogether 108 Asian children aged under 13 years, born since 1965, received treatment for tuberculosis. For each case four controls were selected; they were matched to the case by month and year of birth, sex, and ethnic origin. Cases and controls were all born in Birmingham. Of the cases, 62 out of $108(57 \%)$ had received BCG, and of the controls, 336 out of $432(78 \%)$ had received BCG. The estimated protective efficacy of vaccination was $64 \%$ (95\% confidence limits, $43 \%$ and $77 \%$ ).

Routine BCG vaccination in infant Asians confers useful protection against the development of tuberculosis in childhood.

The school BCG vaccination scheme in England and Wales continues to provide a high degree of protection against the development of tuberculosis in adolescents and young adults. ${ }^{1}$ The annual notification rate for tuberculosis in the white indigenous population, however, has now fallen to 6.9/100 $000^{2}$ and it has therefore been recommended that the scheme be discontinued before 1990 provided that the downward trend in notifications continues. ${ }^{3}$ Children born in the United Kingdom of Asian immigrant parents have a notification rate at least 20 times as high as that in white children, ${ }^{4}$ hence it has also been recommended that newborn Asians and other groups at high risk of developing tuberculosis should continue to receive BCG. ${ }^{3}$ In 1986, 113 health districts in England and Wales routinely offered BCG to newborn Asians (CL Miller, personal communication), but little is known about the effectiveness of these vaccination schemes.

We therefore conducted a study on the effectiveness of BCG vaccination in infant Asians in Birmingham using the case-control method. ${ }^{5}$

\section{Patients and methods}

BCG SCHEME IN BIRMINGHAM

BCG vaccination has been offered routinely to all Asian infant children born in Birmingham since September 1965. This was given to children three months after birth at a central clinic by a doctor and team of health visitors experienced in vaccination technique. Freeze dried vaccine $(0.1 \mathrm{ml}$, Glaxo Laboratories Ltd; 8-26 colony forming units/ ampoule) was injected intradermally over the insertion of the left deltoid muscle using a syringe and needle. In all cases tuberculin testing was performed three days before vaccination. Tuberculin testing was repeated three months after vaccination, except in 1969-70. This was usually performed by Mantoux testing using 10 units of tuberculin purified protein derivative. The result was read after three days and induration of $5 \mathrm{~mm}$ or more when read across the forearm was regarded as a positive result.

From 1979 onwards most vaccinations were given immediately after birth at Birmingham maternity hospitals in order to increase the coverage of the vaccination scheme. The present study was confined to subjects born between 1965 and 1979 who were eligible for BCG vaccination three months after birth under the original scheme and who developed tuberculosis before the age of 13 years.

Asian children reaching the age 13 years are again routinely tuberculin tested under the school BCG scheme and offered vaccination if tuberculin negative.

In 1966, 1440 children were born in Birmingham to parents of Asian or mixed Asian origin. By 1978 this had increased to 2842 ; the average annual number of births over this period was $2304 .^{6}$ 


\section{SELECTION OF CASES}

Cases of tuberculosis in Birmingham were ascertained from the notification register. Details of each patient's place of birth and ethnic origin are included in the notification records. Information was obtained on all Asian children notified up to the end of 1985 who were born in Birmingham after the inception of the BCG scheme. Further clinical details on all patients were obtained by examining the casenotes and radiographs. Where casenotes were unobtainable, details were obtained from the general practice records.

A diagnosis of tuberculosis was accepted if there was bacteriological or histological evidence of tuberculosis, or if there was pulmonary disease without positive bacteriology, or if there were radiological features consistent with tuberculosis that resolved after antituberculous treatment. Lymph node disease was diagnosed on the basis of histology, and in some cases, on the basis of clinical examination if there was persisting lymphadenopathy and a history of close contact with a case of tuberculosis. In all other cases of non-pulmonary disease, a diagnosis of tuberculosis was only accepted if there was a positive bacteriology or histology result.

Notified subjects given antituberculous treatment, whether tuberculin positive or negative, who had no clinical, radiological, or bacteriological evidence of tuberculous disease were considered to have received chemoprophylaxis and were excluded from analysis.

Patients' vaccination histories were obtained from the vaccination records, which are kept in a central filing store. Patients who had received BCG vaccination at birth or at any other time because of known contact with a case of tuberculosis were excluded from the study. Likewise, children notified after the age of 12 years were excluded because of their eligibility for inclusion in the school BCG scheme.

\section{SELECTION OF CONTROLS}

Controls were selected by examination of the vaccination records. They were all subjects born in Birmingham, and they were matched to cases on the basis of month and year of birth, sex, and Asian ethnic origin-only subjects with recognised Asian names were chosen. ${ }^{7}$

The vaccination records are filed according to month and year of birth. The records for individuals born in a particular month are filed in consecutive drawers. Within these drawers, for a given month, records are filed in alphabetical order. Controls were chosen by randomly selecting one of the drawers containing the records corresponding to the month and year of birth of a given case. Within a selected drawer the first four records that fulfilled the matching criteria were examined, this meant that for each case information on four individually matched controls was obtained.

Subjects who had received BCG at any time other than under the scheme for newborn Asians were excluded.

STATISTICAL ANALYSIS

The relative risk of contracting tuberculosis in the vaccinated group compared with the risk in the unvaccinated group was estimated by calculation of the odds ratio $(\psi)^{8}$ and appropriate confidence limits. ${ }^{9}$ The 'protective efficacy' of vaccination and the percentage reduction in cases of tuberculosis in the BCG vaccinated group compared with the unvaccinated group is given by $(1-\psi) 100 .^{5}$

The proportion of potential new cases of tuberculosis in Asian children prevented, the "prevented fraction' ${ }^{10}$ is given by $\rho(1-\psi) / \rho(1-\psi)+\psi$, where $\rho$ is the proportion of cases given $\mathrm{BCG}$.

Calculations were performed using published programmes. ${ }^{11}$

\section{Results}

The total number of tuberculosis notifications up to the end of 1985, for Asians born in Birmingham between September 1965 and December 1978, was 267. Most patients were followed up at the Birmingham Chest Clinic; the others were treated at four other hospitals within Birmingham. From this total, $159(60 \%)$ subjects were excluded from the analysis for the following reasons: $113(42 \%)$ were given chemoprophylaxis only, $25(9 \%)$ were older than 12 years, $14(5 \%)$ were given BCG because of contact with a case of tuberculosis, and one patient died at two months of disseminated tuberculosis discovered on postmortem examination before being eligible to receive vaccination. Six subjects $(2 \%)$ were excluded from analysis because either clinical details or information on vaccination states were not available.

Of the 108 cases included in the final analysis, 47 $(44 \%)$ were boys and $61(56 \%)$ girls; 59 (55\%) were of Indian ethnic origin, and $49(45 \%)$ were of Pakistani or Bangladeshi origin. Respiratory disease only was present in $79(73 \%)$, non-respiratory disease only in $22(20 \%)$, and both respiratory and non-respiratory in seven ( $7 \%$ ). Altogether 21 samples gave a positive culture, mainly from gastric washings; seven of these samples were smear positive. Sites of disease are given in table 1.

The principle findings are shown in table 2 . Of the cases the reason that the vaccination had not been given was unrecorded in 31 children, 15 others 
defaulted. In the controls, the reason that BCG had not been given was unrecorded in 73 children; 22 others defaulted, and one refused.

For analysis the results were expressed in terms of the vaccination state of each matched set of four controls (table 3 ). The estimated protective efficacy for the vaccine was $64 \%$ with $95 \%$ confidence limits of $43 \%$ and $77 \%$. The prevented fraction was 0.50 -that is, BCG prevented 50 out of every 100 potential new cases of tuberculosis.

In all cases and controls tuberculin testing before vaccination gave a negative result. Thirty six cases had postvaccination tuberculin testing performed.

Table 1 Site of disease (No of cases $=108$ )

\begin{tabular}{lr}
\hline Respiratory disease (radiographic assessment) & \\
Enlarged nodes only & 34 \\
Pulmonary lesion with enlarged nodes & 22 \\
Pulmonary lesion only & 17 \\
Pulmonary and pleural & 3 \\
Pleural only & 1 \\
Normal (positive gastric washings) & 2 \\
Non-respiratory & \\
Lymph nodes & 9 \\
Miliary & 5 \\
Bone and joint & 3 \\
Peritoneal & 2 \\
Bowel and urinary tract & 1 \\
Meninges & 1 \\
Conjunctival & 1 \\
Respiratory and non-respiratory & \\
(site of non-respiratory disease given) & \\
Lymph nodes & \\
lleocaecal & 5 \\
Dactylitis & 1 \\
\hline
\end{tabular}

Table 2 No (\%) of cases and controls given BCG vaccination

\begin{tabular}{lcc}
\hline & Cases & Controls \\
\hline No given BCG (\%) & $62(57)$ & $336(78)$ \\
No not given BCG (\%) & $46(43)$ & $96(22)$ \\
\hline Total & 108 & 432 \\
\hline
\end{tabular}

Table 3 Sets of four controls classified according to number vaccinated and to vaccination state of matched case

\begin{tabular}{llllllll}
\hline & \multicolumn{2}{l}{$\begin{array}{l}\text { No of controls (per set of four) } \\
\text { given } B C G\end{array}$} \\
\cline { 2 - 7 } & 0 & 1 & 2 & 3 & 4 & Total \\
\hline $\begin{array}{c}\text { Sets matched to } \\
\text { vaccinated cases }\end{array}$ & 0 & 0 & 12 & 23 & 27 & 62 \\
$\begin{array}{c}\text { Sets matched to } \\
\text { unvaccinated cases }\end{array}$ & 0 & 3 & 12 & 16 & 15 & 46 \\
\hline
\end{tabular}

In $31(86 \%)$ the result was positive; three were positive when retested and two were revaccinated. The mean (SD) diameter of the Mantoux reactions in those cases vaccinated was $8.7(2 \cdot 1) \mathrm{mm}$. Postvaccination tuberculin testing was done in 193 controls. In $184(95 \%)$ the result was positive; seven others were positive when retested, and two defaulted. The mean (SD) diameter of postvaccination tuberculin reactions in the controls was $9.4(2.7)$ $\mathrm{mm}$. This was not significantly different from the mean postvaccination reactions in the cases who had been vaccinated (normal deviate test).

\section{Discussion}

There are little data on the effectiveness of BCG vaccination in the newborn in the United Kingdom. In Manchester, 10326 neonates were vaccinated at a group of maternity hospitals between 1951-60; 15152 babies born at the same hospitals did not receive vaccination. Over the same period 42 children born in the same hospitals developed tuberculosis: all of these cases came from the unvaccinated group. ${ }^{12} \mathrm{~A}$ follow up study in children born between 1965-80 showed that BCG gave a protective effect of over $75 \% .^{13}$

Similarly, there are little data from studies based in the United Kingdom on the value of BCG vaccination in Asian subjects. Evidence that BCG vaccination protects against tuberculosis in Asian subjects comes from a contact study ${ }^{14}$ : seven out of $425(2 \%)$ contacts of patients with tuberculosis who were vaccinated developed tuberculosis compared with 57 out of $1479(4 \%)$ unvaccinated contacts.

Recent studies on the efficacy of BCG in the newborn, mainly sponsored by the World Health Organisation, gave results similar to ours and showed a protective efficacy of between $53 \%$ and $74 \% .{ }^{15-18}$ These agreed with earlier studies on BCG vaccination in the newborn, which consistently showed a beneficial effect, ${ }^{19}{ }^{20}$ but are in marked contrast to the widely divergent results of prospective BCG trials in adolescents and young adults in several countries, some of which showed negative results. ${ }^{21} 22$

Case-control methods to evaluate the effectiveness of BCG vaccination are quicker and less costly than are prospective methods, but they are more susceptible to bias. ${ }^{5}$ Control subjects in the present study were not clinically examined, therefore the presence of tuberculosis in some of these subjects cannot be excluded. Most cases of tuberculosis in childhood, however, arise as a result of close contact with an infected adult, and under the contact tracing system in Birmingham children in contact with known adult cases will have been examined. 
Although all the control subjects were of Asian origin, they were not individually matched according to whether the matched case was of Pakistani or Bangladeshi or of Indian origin. Additionally, they were not individually matched according to socioeconomic state as this information was not available from the vaccination records. A wide disparity in the proportion of cases and controls coming from different ethnic and social backgrounds could have biased the results if the coverage of the scheme differed in any important respect between ethnic and social groups within the Asian community. It was noted that cases and controls, however, came from similar districts within Birmingham, and these tend to be ethnically and socially homogeneous. The method of selection of controls should have prevented a preponderance of subjects from any particular social or ethnic grouping.

A study carried out in Birmingham, when neonatal BCG vaccination of newborn Asians was first introduced into Birmingham maternity hospitals in 1979 , showed that only $69(46 \%)$ out of 149 children were Mantoux positive after 22 months. ${ }^{23}$ The authors suggested that this result could be explained by reduced cell mediated immunity in neonates. A similar study from Bradford showed that $59(45 \%)$ out of 131 Asian babies given BCG within the first three months of birth gave negative or grade 1 Heaf reactions when tested between the ages of 3 months and 2 years. ${ }^{24}$ Conflicting results from another centre in the United Kingdom showed that 353 $(98 \%)$ out of 361 Asian neonates given BCG were tuberculin positive when tested three months later ${ }^{25}$; these results accord with those of the present study. If impaired immunity was the cause of diminished tuberculin sensitivity after vaccination then a fairly constant proportion of Asian children would be expected to be non-reactors to tuberculin after vaccination. A more likely explanation for the foregoing results is variation in the quality of vaccine administration both within and between different centres in the United Kingdom.

In the 1978-9 national survey of tuberculosis in England and Wales, $23 \%$ of the notified cases of childhood tuberculosis were receiving chemoprophylaxis ${ }^{4}$ compared with $42 \%$ in the present study. There are two possible explanations for our higher figure. Firstly, physicians in Birmingham may have more readily notified children receiving chemoprophylaxis than physicians elsewhere. Secondly, because we had access to patients' casenotes and full sets of radiographs, it is possible that we were better able to distinguish cases of tuberculosis from children given chemoprophylaxis; in the 1978-9 national survey, assessment was based only on a single pretreatment chest radiograph and on information provided by the notifying physician.

Most of the children given chemoprophylaxis were treated solely on the basis of a positive tuberculin test, which may have been induced by previous BCG vaccination. Inclusion of these subjects in our analysis would have resulted in a reduction in the observed effect of the vaccine.

This study has shown that routine BCG vaccination in infant Asians in Birmingham confers useful protection against the subsequent development of tuberculosis in childhood. Further studies are required to assess the overall value of routinely vaccinating Asian newborn children, taking into account not only the efficacy of the vaccine, but also the declining incidence of tuberculosis in the Asian community in the United Kingdom, ${ }^{2}$ the cost of treatment for tuberculosis, and the loss of tuberculin testing as a diagnostic tool once BCG has been given.

We thank Dr VH Springett, Dr N Gill, and Ms L Fernandez for their advice, and doctors in Birmingham for giving us access to details of patients under their care. We also thank Mrs EH Armstrong of the University of Birmingham Department of Social Medicine, and Mrs V Stanley for their help.

\section{References}

1 Sutherland I, Springett VH. Effectiveness of BCG vaccination in England and Wales in 1983. Tubercle 1987;68:81-92.

${ }^{2}$ Medical Research Council Tuberculosis and Chest Diseases Unit. National survey of notifications of tuberculosis in England and Wales in 1983. Br Med $J$ 1985;291:658-61.

${ }^{3}$ Department of Health and Social Security. The school BCG vaccination programme. London: DHSS, 1985. (DA (85) 27.)

${ }^{4}$ Medical Research Council Tuberculosis and Chest Diseases Unit. Tuberculosis in children in a national survey of notifications in England and Wales, 1978-79. Arch Dis Child 1982;57:734-41.

5 Smith PG. Retrospective assessment of the effectiveness of BCG vaccination against tuberculosis using the case-control method. Tubercle 1982;62:23-35.

${ }^{6}$ Neale AB, Haine J. Birmingham Statistics. Vol 11-22. Birmingham City Council Central Statistical Office.

${ }^{7}$ Henley A. Asian patients in hospital and at home. Bath: Pitman Medical Publishing Company, 1979:87-104.

${ }^{8}$ Miettinen OS. Estimation of relative risk from individually matched series. Biometrics 1970;26:75-86.

${ }^{9}$ Miettinen OS. Estimability and estimation in case-referent studies. Am J Epidemiol 1976;103:226-35.

11) Miettinen OS. Proportion of disease caused or prevented by a given exposure, trait or intervention. Am $J$ Epidemiol 1974;99:325-32.

"Rothman KJ, Boice JD. Epidemiologic analysis with a programmable calculator. 2nd ed. Boston: Epidemiology Resources Inc, 1983.

12 Griffiths MI. BCG vaccination in infancy. Health and Tuberculosis Conference 1962. Transactions of the Sixth Commonwealth Conference organised by the Chest and Heart Association. London: Chest and Heart Association, 1962:47-52.

13 Curtis HM, Leck I, Bamford FN. Incidence of childhood tuberculosis after neonatal BCG vaccination. Lancet 1984;i: $145-8$. 
14 Contact Subcommittee of the Research Committee of the British Thoracic Association. A study of a standardised contact procedure in tuberculosis. Tubercle 1978;59:245-59.

15 Anonymous. Tuberculosis control programme and expanded programme on immunisation. Efficacy of infant BCG vaccination. Weekly Epidemiological Review 1986;28:216-18.

16 Padungchan S, Konjanart S, Kasiratta S, Daramas S, ten Dam HG. The effectiveness of BCG vaccination of the newborn against childhood tuberculosis in Bangkok. Bull WHO 1986;64:247-58.

17 Tidjani O, Amedome A, ten Dam HG. The protective effect of $B C G$ vaccination of the newborn against childhood tuberculosis in an African community. Tubercle 1986;67:269-81.

18 Kue Young T, Hershfield ES. A case-control study to evaluate the effectiveness of mass neonatal BCG vaccination among Canadian Indians. Am J Public Health 1986;76:783-6.

19 ten Dam HG, Hitze KL. Does BCG vaccination protect the newborn and young infants? Bull WHO 1980;58:37-41.
20 Anonymous. BCG vaccination in the newborn [Editorial]. $\mathrm{Br}$ Med $J$ 1980;281:1445-6.

21 Anonymous. Is BCG effective? [Editorial]. Tubercle 1981; 62:219-21.

22 Clemens JD, Chuong JJH, Feinstein AR. The BCG controversy: a methodological reappraisal, JAMA 1983;249:2362-9.

${ }^{23}$ Grindulis H, Baynham MID, Scott PH, Thompson RA, Wharton BA. Tuberculin response two years after BCG vaccination at birth. Arch Dis Child 1984;59:614-9.

${ }^{24}$ Lumb KM, Bandaranayake R, Bevan PJ. BCG vaccination in infancy. Public Health 1986;100:54-5.

${ }^{25}$ Hadfield JW, Allen J, Windebank WJ. Sensitivity of neonates to tuberculin after BCG vaccination. Br Med J 1986;292:989-91.

Correspondence to Dr GE Packe, Department of Respiratory Physiology, East Birmingham Hospital, Bordesley Green East, Birmingham B9 5ST.

Accepted 14 October 1987 